\title{
Pode a agricultura urbana contribuir para o desenvolvimento de cidades e comunidades sustentáveis?
}

\author{
Can urban agriculture contribute to the development of sustainable cities and communities? \\ ¿Puede la agricultura contribuir al desarrollo de ciudades y comunidades sostenibles?
}

Recebido: 11/04/2021 | Revisado: 18/04/2021 | Aceito: 23/04/2021 | Publicado: 08/04/2021

\author{
Moisés Savian \\ ORCID: https://orcid.org/0000-0002-1672-659X \\ Universidade do Estado de Santa Catarina, Brasil \\ E-mail: moisessavian@gmail.com \\ Pedro Boff \\ ORCID: https://orcid.org/0000-0002-9041-5503 \\ E-mail: boff.pedro@yahoo.com.br \\ Mari Inês Carissimi Boff \\ ORCID: https://orcid.org/0000-0003-1700-8837 \\ Universidade do Estado de Santa Catarina, Brasil \\ E-mail: mari.boff@udesc.br
} Empresa de Pesquisa Agropecuária e Extensão Rural de Santa Catarina, Brasil

\begin{abstract}
Resumo
Que a agricultura é praticada nas cidades é um fato, porém poucos estudos tem a finalidade de compreender esse fenômeno e as suas contribuições para o desenvolvimento socioambiental. Objetivou-se analisar a contribuição da agricultura urbana no desenvolvimento sustentável das cidades e comunidades. A metodologia consistiu na análise de artigos científicos publicados em periódicos indexados, entre o período de 2009 e 2019, que tinham como foco o estudo da agricultura urbana no Brasil. Foram acessados 667 artigos, dos quais 40 atenderam critérios de inclusão. Os estudos selecionados foram desenvolvidos em 12 estados brasileiros e envolveram 123 pesquisadores. Os resultados demonstram que a agricultura urbana é realizada em todas as regiões do Brasil, apresenta características multifuncionais diferenciadas, possui papel importante na manutenção da agrobiodiversidade e auxilia na segurança alimentar e nutricional. Além disso a agricultura urbana é um componente importante nas relações econômicas, sociais e ecológicas das cidades e pode gerar benefício aos espaços urbanos, promover a saúde e auxiliar nos processos educacionais. Concluímos que a agricultura urbana tem potencialidade para ser adotada como uma estratégia no desenvolvimento de cidades e comunidades sustentáveis.
\end{abstract}

Palavras-chave: Agroecologia; Cidades sustentáveis; Cultivo urbano; Horticultura urbana.

\begin{abstract}
That agriculture occurs in cities is a fact, but few studies have the purpose of understanding this phenomenon and its contributions to socio-environmental development. The objective was to analyze the contribution of urban agriculture to the sustainable development of cities and communities. The methodology consisted of analyzing scientific articles published in indexed journals, between the period of 2009 and 2019, which focused on the study of urban agriculture in Brazil. 667 articles were accessed, of which 40 met inclusion criteria. The selected studies involve 12 Brazilian states and 123 researchers. The results demonstrate that urban agriculture occurs in all regions of Brazil, has different multifunctional characteristics, plays an important role in maintaining agrobiodiversity and contributes to food and nutritional security. Furthermore, urban agriculture is an important component in the economic, social and ecological relations of cities and can generate benefits for urban spaces, promote health and assist in educational processes. We conclude that urban agriculture has the potential to be adopted as a strategy in the development of sustainable cities and communities.
\end{abstract}

Keywords: Agroecology; Sustainable cities; Urban cultivation; Urban horticulture.

\section{Resumen}

Que la agricultura se practica en las ciudades es un hecho, pero pocos estudios tienen el propósito de comprender este fenómeno y sus aportes al desarrollo socioambiental. El objetivo fue analizar la contribución de la agricultura urbana al desarrollo sostenible de ciudades y comunidades. La metodología consistió en analizar artículos científicos publicados en revistas indexadas, entre el período 2009 y 2019, que se enfocaron en el estudio de la agricultura urbana en Brasil. Se consultaron 667 artículos, de los cuales 40 cumplieron criterios de inclusión. Los estudios seleccionados se desarrollaron en 12 estados brasileños e involucraron a 123 investigadores. Los resultados demuestran que la agricultura urbana se lleva a cabo en todas las regiones de Brasil, tiene diferentes características multifuncionales, juega un papel 
importante en el mantenimiento de la agrobiodiversidad y ayuda en la seguridad alimentaria y nutricional. Además, la agricultura urbana es un componente importante en las relaciones económicas, sociales y ecológicas de las ciudades y puede generar beneficios para los espacios urbanos, promover la salud y ayudar en los procesos educativos. Concluimos que la agricultura urbana tiene potencial para ser adoptada como estrategia en el desarrollo de ciudades y comunidades sostenibles.

Palabras clave: Agroecología; Ciudades sostenibles; Cultivo urbano; Horticultura urbana.

\section{Introdução}

A existência do Homo sapiens é estimada em cerca de 200.000 anos, no entanto a prática da agricultura começou somente a cerca de 12.000 anos (Harari, 2017). Nos assentamentos em grandes comunidades do Neolítico (10.000 a 6.000 a.C.) a sobrevivência foi viabilizada pela coleta e pela caça, já que a transição para a agricultura e a criação de animais aconteceu aos poucos (Gondim, 2014). A agricultura como ato de cultivar e criar é a atividade mais antiga em que os seres humanos modificam a natureza e sua adoção levou a transformação de grandes extensões de terra do planeta em área de lavouras e pastagens (Dale et al., 2013).

A agricultura moderna surge nos séculos XVIII e XIX em diversas áreas da Europa o que possibilitou o desenvolvimento de uma produção agrícola que viabilizasse o fim da escassez crônica de alimentos (Veiga, 2007). Com diversas transformações a agricultura foi capaz de produzir um excedente comercializável para suprir a demanda de uma população não agrícola, viabilizando o progresso industrial e urbano (Mazoyer \& Roudart, 2010). No entanto, em consequência do sistema econômico cerca de 690 milhões de pessoas ainda estão subnutridas no mundo, grande parte vivendo no espaço rural (FAO et al., 2020; Mazoyer \& Roudart, 2010).

Como reflexo do aprimoramento da agricultura e do aumento da produtividade agrícola o século XX foi marcado pela expansão das cidades. A população urbana mundial que em 1950 era de 750 milhões de pessoas cresceu para 3,6 bilhões em 2011 (ONU, 2012). Projeções estimam que até 2050 teremos cerca de 9,7 bilhões de habitantes vivendo no planeta e espera-se que as áreas urbanas absorvam praticamente todo o crescimento da população mundial (ONU, 2019). O crescimento da população fará da urbanização uma das tendências mais transformadoras no século XXI (ONU, 2017).

No Brasil a expansão do espaço urbano ocorreu sem preocupação com as questões ambientais gerando diversos problemas como o desmatamento, escassez de áreas verdes, erosão, excessiva impermeabilização do solo, saneamento básico, e resíduos sólidos (Silva \& Travassos, 2008). Devido à falta de planejamento urbano a maioria das cidades brasileiras enfrentam ameaças sem precedentes reflexo do padrão insustentável de produção e consumo, a poluição e as mudanças climáticas (ONU, 2017).

A agricultura urbana é uma estratégia para atender a segurança alimentar e nutricional e promover o desenvolvimento local melhorando o ambiente urbano com a ampliação da biodiversidade, manutenção dos serviços ambientais e incremento de áreas verdes (FAO, 2014). Por possuir um caráter multifuncional a agricultura urbana pode contribuir ainda na preservação da cultura alimentar, na gestão dos resíduos, na conservação da biodiversidade, no controle do microclima, na geração de trabalho, na saúde humana e educação (Lovell, 2010). As experiências de agricultura urbana orientadas pelos princípios da Agroecologia, trazem possibilidades para transformar a vida das pessoas e das comunidades com a produção de alimentos saudáveis e a conservação dos recursos naturais (Moura et al., 2013). Esse trabalho teve como objetivo analisar as possibilidades da agricultura urbana contribuir para o desenvolvimento de cidades e comunidades sustentáveis. 


\section{Metodologia}

A revisão bibliográfica sistemática foi realizada com base na literatura científica disponível na Plataforma da Coordenação de Aperfeiçoamento de Pessoal de Nível Superior (CAPES). ${ }^{1}$ A Plataforma foi escolhida pela possibilidade de abranger periódicos brasileiros que não estariam indexados em bases de dados internacionais. O extrato incluiu publicações completas publicadas no período de 01 de janeiro de 2009 à 31 de dezembro de 2019. Para atender os objetivos do trabalho foi utilizada a palavra-chave de busca "agricultura urbana", associada a "Brasil", em inglês, português e espanhol.

A seleção dos artigos foi baseada na metodologia PRISMA (Preferred Reporting Items for Systematic Reviews and Meta-Analyses) apresentada por Urrútia e Bonfill (2010). Esta metodologia estabelece que os artigos encontrados na pesquisa serão selecionados a partir do resumo com base nos critérios de elegibilidade (inclusão ou exclusão). Foram incluídos artigos que tiveram como tema central de pesquisa a agricultura urbana e foram realizados no Brasil. Buscou-se somente artigos científicos publicados em periódicos com revisão por pares. Editoriais de revistas, artigos publicados em revistas de divulgação científica, artigos de revisão ou que não apresentam dados primários de pesquisa foram excluídos. Os artigos selecionados foram lidos na íntegra e compõem as referências utilizadas para as análises desse artigo.

\section{Resultados}

A consulta a base de dados da Plataforma CAPES resultou na seleção de 667 artigos. Do total 79,3\% (529) foram excluídos por não se adequarem ao tema, uma vez que os artigos não tinham como objeto central a agricultura urbana. Outros 10,5\% (70) foram excluídos por não serem pesquisas realizadas no Brasil. Dez publicações (1,5\%) por não apresentarem dados primários e 2,7\% (18) por outros fatores ${ }^{2}$. Assim, após análise da elegibilidade restaram 40 artigos para o estudo. A Figura 1 apresenta os resultados obtidos a partir da estratégia de busca.

Referente ao idioma 55,0\% (22) artigos foram publicados em português, 42,5\% (17) em inglês e 2,5\% (1) em espanhol. Considerando a distribuição ao longo do tempo a publicação de artigos sobre agricultura urbana variou entre 1 a 9 artigos por ano, resultando numa média de 3,6 artigos por ano. A maior parte dos artigos (67,5\%) foram publicados entre 2015 e 2019 , totalizando 5,4 artigos por ano, o que indica um aumento na produção científica sobre o assunto.

Os artigos foram publicados em 34 periódicos científicos nacionais e internacionais. A maioria dos periódicos tiveram um artigo publicado. Foram encontradas duas publicações nos periódicos Ambiente \& Sociedade, Boletim do Museu Paraense Emilio Goeldi, Environmental Pollution e Saúde e Sociedade e três no periódico Demetra: Food, Nutrition e Health. Contribuíram para os artigos 123 autores de instituições brasileiras e internacionais. Desses, 15 participaram de mais de um artigo e teve um predomínio $(81 \%)$ de autores pertencentes a universidades e instituições de pesquisa brasileiras.

\footnotetext{
${ }^{1}$ A Plataforma da CAPES é mantida pelo Ministério da Educação do Brasil para subsidiar os pesquisadores brasileiros e conta com um acervo de 1.419 periódicos e mais nove bases referenciais de todas as áreas do conhecimento.

${ }^{2}$ Foram excluídos editoriais de revistas científicas, artigos publicados em revistas de divulgação científica e trabalhos que apresentaram duplicidade.
} 
Figura 1. Estratégia de busca da produção bibliográfica sobre agricultura urbana no Brasil entre os anos 2009 a 2019 e resultados encontrados na revisão sistemática.

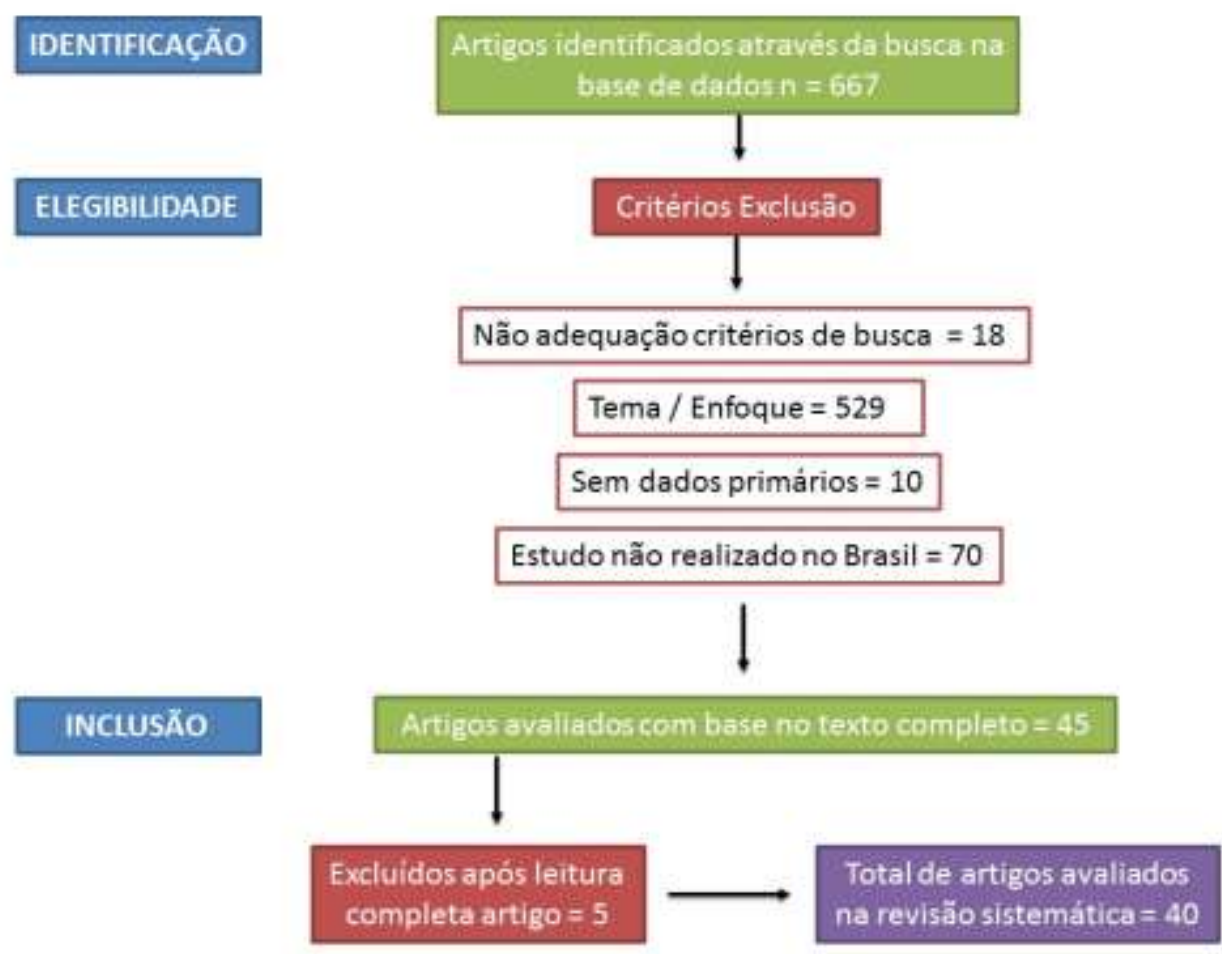

Fonte: Autores.

As pesquisas que resultaram nas publicações foram desenvolvidas em 12 estados brasileiros. O estado de São Paulo lidera com doze publicações, seguido do Pará e Minas Gerais com seis e cinco publicações respectivamente (Tabela 1). Duas pesquisas foram desenvolvidas baseadas na realidade de todo o país, enquanto, uma não mencionou especificamente a cidade onde o estudo foi desenvolvido. Em quatro pesquisas internacionais uma cidade brasileira foi analisada em estudo comparativo.

Tabela 1. Distribuição das publicações no período entre 2009 a 2019 sobre agricultura urbana no Brasil por estado da federação, município e número de artigos.

\begin{tabular}{ll}
\hline Estados da Federação & \multicolumn{1}{c}{ Município (no de artigos) } \\
\hline Acre & Rio Branco (2) \\
\hline Alagoas & Maceió (1) \\
\hline Amazonas & $\begin{array}{l}\text { São Gabriel da Cachoeira, Santa Isabel do Rio Negro e } \\
\text { Barcelos (1) }\end{array}$ \\
\hline Goiás & Santo Antônio do Descoberto (1) \\
\hline Maranhão & Imperatriz (1) \\
\hline Minas Gerais & Belo Horizonte (4), Viçosa (1) \\
\hline Pará & Belém (2), Benevides (1), Castanhal (1), Santarém (1), 14 \\
Paraná & Maricípios Noroeste Paraense (1) \\
\hline Pernambuco & Recife (2) \\
\hline Rio Grande do Norte & Natal (1) \\
\hline Rio de Janeiro & Rio de Janeiro (3) \\
\hline São Paulo & Embu das Artes (7), Rio Claro (1), São Carlos (1) e São Paulo \\
& $(3)$ \\
\hline
\end{tabular}

Fonte: Autores. 
Na metodologia da pesquisa utilizada nos artigos predominam as abordagens qualitativas usando uma ou mais técnicas de pesquisa como entrevistas, aplicação de questionários, análise documental, realização de oficinas e grupo focais, análise histórica e observação direta ou participativa. Em 55\% dos artigos foi utilizado a entrevista (semiestruturada ou em profundidade) para a coleta de dados, em $20 \%$ os dados foram coletados com a utilização de questionário e em 17,5\% houve a análise do número e espécies de plantas ocorrentes em quintais ou hortas urbanas. As pesquisas que utilizaram metodologias que envolvem análises em laboratório ou geotecnologias como o geoprocessamento de imagens ocorreram em apenas três artigos (7,4\% do total).

\section{Discussão}

As distintas abordagens conferidas ao tema da agricultura urbana demonstram o caráter multidisciplinar das pesquisas. A partir da análise dos artigos foi possível classificar os resultados e sistematizar as informações com base em três temas: (1) Características da agricultura urbana brasileira; (2) Agricultura urbana, saúde e educação; (3) Agricultura e desenvolvimento urbano. Os temas identificados são apresentados e analisados na sequência, considerando as informações publicadas pelos autores.

\subsection{Características da Agricultura Urbana Brasileira}

Pela análise das publicações verificou-se que a agricultura urbana (AU) é uma prática presente em diversas cidades brasileiras. Em Natal (RN), uma porcentagem significativa de famílias desenvolve atividades agrícolas no urbano (Locatel \& Azevedo, 2010), assim como em Maceió (AL), onde a ocorrência de cultivos e criações de animais no espaço urbano refletem a existência de "micros cenários do agrícola no urbano" (Santos, 2017). Embora a literatura acessada relate a ocorrência da AU nas distintas regiões do país não existem dados que quantifiquem a proporção da população que pratica essa atividade.

A AU ocorre de distintas formas e com características diferenciadas. Praticada, em geral, em pequenos espaços no urbano, como quintais, terrenos baldios, terrenos íngremes, margens de vias pouco movimentadas, entre outros lugares (Santos, 2017). Em quintais domésticos onde o espaço é limitado o cultivo é realizados em vasos (Batitucci et al., 2019). Em Belém (PA), Wandscheer e Medeiros (2012) observaram espaços cultivados tanto no centro quanto na periferia do território urbano e destacaram diferenças no tamanho do cultivo de acordo com a localização. Já em Belo Horizonte (MG), a AU é realizada em áreas urbanas periféricas, caracterizadas pela baixa renda dos moradores e pelo déficit de acesso aos serviços e infraestrutura urbana, o que representa uma forma possível de produção e reprodução da vida (Araújo, 2016). Ao longo do rio Negro, no estado do Amazonas, a prática da agricultura é difundida nas cidades e conta com a importante participação de populações indígenas, sendo a produção agrícola destinada principalmente à subsistência familiar, com uma pequena parte do excedente comercializado (Emperaire \& Eloy, 2015). No Pará, foram identificadas categorias distintas de agricultores, tendo os que desenvolvem a atividade como principal fonte de renda, servidores públicos que praticam a agricultura de maneira complementar ao seu ofício e comerciantes que realizam agricultura urbana paralelamente ao seu comércio (Rodrigues et al., 2020).

A motivação para a prática da agricultura nas cidades brasileiras está relacionada aos benefícios que a atividade promove. Em Belo Horizonte os entrevistados apontaram como principais motivações a segurança alimentar e nutricional conjugada a outros fatores como: o envolvimento da comunidade vizinha na atividade, o aumento da renda, a limpeza dos lotes e a oportunidade de exercer, através das hortas, uma atividade de lazer (Araújo, 2016). A produção de alimentos sem agrotóxicos e o papel terapêutico das hortas urbanas principalmente para os mais idosos foi destacado em Maceió (Santos, 2017). Em Rio Branco o cultivo de plantas alimentares em quintais urbanos auxilia no tratamento de doenças e promove a conservação da agrobiodiversidade, bem-estar aos moradores pela melhoria da paisagem, ambiência microclimática e espaço de lazer (Siviero et al., 2011). 
As hortas urbanas produzem alimentos para o autoconsumo, para a comercialização e contribuem no alcance da segurança alimentar e nutricional. Nos municípios da Amazônia, Emperaire e Eloy (2015), afirmam que nas hortas urbanas são produzidos alimentos destinados para o autoconsumo e/ou para o mercado informal e as vendas ocorrem na rua ou mediante solicitação. Ao analisarem a dieta de agricultores urbanos em Rio Claro, Eichemberg e Amorozo (2013) relataram que dos 106 itens consumidos, 26,4\% (28) foram produzidos em hortas caseiras, havendo predominância no cultivo e produção de frutas. Villares de Carvalho e Nella Branduini (2017) registraram que em Recife, nordeste brasileiro, 38\% da produção alimentar urbana é utilizada para autoconsumo. Para as famílias socialmente desfavorecidas no Brasil, as hortas urbanas podem complementar suas dietas, fornecendo à família uma variedade de alimentos saudáveis e nutritivos (de Medeiros et al., 2018). Aliadas as políticas públicas as hortas urbanas possuem uma importância ímpar na efetivação do direito humano a alimentação, proporcionando alimentos saudáveis, de qualidade e acessível à comunidade (Siqueira et al., 2019).

A responsabilidade pelo cultivo nas hortas urbanas pode variar entre os gêneros e os trabalhos apontam para o predomínio da mulher. Em Rio Branco, Siviero et al. (2012) afirmam que a responsabilidade pela manutenção dos quintais urbanos é das mulheres, notadamente aquelas com idade superior a 50 anos. Nas pesquisas de Emperaire e Eloy (2015) foram entrevistados 30 pessoas, dentre as quais 29 eram mulheres, concluindo que elas desempenham um papel central no manejo e conservação da agrobiodiversidade. Contrastando com essas conclusões, em Benevides, Pará, a produção comercial de flores e plantas ornamentais realizada em regiões urbanas e periurbanas é uma atividade predominantemente masculina (Abud et al., 2019).

A agrobiodiversidade foi tema considerado em vários artigos e sempre associada as múltiplas finalidades do cultivo de plantas, em especial aos usos alimentar, ornamental e medicinal. Em Santarém foram identificados 25 jardins com 225 espécies alimentares, ornamentais, medicinais e para produção de borracha (WinklerPrins \& Oliveira, 2010) enquanto que em Rio Claro (SP), 98 espécies alimentares, com média de 21 por horta (Eichemberg \& Amorozo, 2013). Já em Rio Branco, 77 espécies alimentares distribuídas entre 34 famílias botânicas (Siviero et al., 2011) e 83 espécies de uso medicinal (Siviero et al., 2012). Em diversos municípios do estado do Pará foram registradas 108 espécies de plantas arbóreas e palmeiras de 40 famílias botânicas diferentes (Rayol et al., 2017). A diversidade genética ocorre também na própria espécie, pois segundo Emperaire e Eloy (2015), foram registradas 106 variedades de mandioca no estado do Amazonas.

A riqueza das espécies pode estar relacionada a alimentação, aos usos das plantas e as motivações e características dos agricultores urbanos. A variação de plantas comestíveis cultivadas em jardins urbanos é importante para a dieta das famílias (de Medeiros et al., 2018). Para Emperaire e Eloy (2015) a agrobiodiversidade é o resultado do interesse constante em novas variedades e da circulação de mudas ou sementes na rede social de agricultores, parentes, vizinhos ou conhecidos, e que a manutenção desses recursos depende desse interesse, combinados com um processo contínuo de inovação e experimentação. Siviero et al. (2012) observaram ainda que a diversidade de espécies está relacionada ao tempo de moradia e a idade dos moradores, afirmando que, a partir dos casos estudados, a probabilidade de ocorrência de espécies é 3 vezes maior em quintais manejados por moradores com idade superior a 50 anos.

Quanto a utilização de agrotóxico os resultados demonstram que a agricultura nas cidades brasileiras não é necessariamente agroecológica. Em Recife (PE) o controle de insetos, através dos inseticidas sintéticos, foi considerado o principal método empregado na agricultura urbana (Moraes et al., 2017) enquanto que em Belém os agricultores raramente utilizam agrotóxicos (Wandscheer \& Medeiros, 2012). A literatura consultada demonstrou que os aspectos relacionados as técnicas de cultivo de plantas em espaços urbanos são pouco abordados necessitando de mais pesquisas com esse objetivo.

A relação entre políticas públicas e AU também é relatada nos artigos. Castelo Branco et al., (2011) analisam o projeto desenvolvido em Santo Antônio do Descoberto (GO), onde a Prefeitura disponibilizou um terreno abandonado para implantação de uma horta urbana. Neste local, 25 famílias cultivavam em lotes individuais de $300 \mathrm{~m}^{2}$ mais de 20 tipos de hortaliças. A renda 
obtida pelas famílias através da comercialização das hortaliças era bastante variável, algumas famílias chegaram a obter um salário mínimo por mês.

O fortalecimento de políticas públicas pode contribuir para o desenvolvimento da AU. Na cidade do Rio de Janeiro, por exemplo, o projeto Hortas Cariocas contou com apoio inicial do governo e demonstrou ser possível criar negócios viáveis e sistemas alimentares saudáveis, integrando assim a viabilidade comercial com responsabilidade social e ecológica (Hearn, 2018). Em Belo Horizonte, Cabannes (2012) relatou a necessidade de investimento em infraestruturas para produção, a aquisição de insumos agrícolas urbanos e o apoio técnico como as principais necessidades dos agricultores urbanos, questões estas que poderiam ser aportadas pelas políticas públicas.

\subsection{Agricultura Urbana, Saúde e Educação}

Os artigos analisados mostram que a agricultura urbana está associada aos temas de saúde e educação, pois promovem a saúde, a educação alimentar e nutricional, incentivam a implantação de hortas escolares e reduzem a contaminação de alimentos através do cultivo sem agrotóxicos. Para ilustrar, a associação dos temas, acima mencionados a implementação do projeto "Colhendo Sustentabilidade: práticas comunitárias de segurança alimentar e agricultura urbana" ${ }^{3}$ realizada no município de Embu das Artes, em São Paulo, agregou fatores sociais, ambientais e econômicos visando sempre a saúde dos membros da comunidade.

A promoção da saúde é um conjunto de estratégias e formas de obter saúde, no âmbito individual e coletivo, que visa atender as necessidades sociais de saúde e garantir a melhoria da qualidade de vida da população (Malta et al., 2016). Baseado nesse conceito, Ribeiro et al. (2015) defendem que os projetos de agricultura urbana agroecológica podem ser considerados como uma ferramenta promotora de saúde, pois contribui para que o ser humano se reconheça como parte do meio ambiente, resgatando o sentimento de pertença e integrando-o socialmente. Além disso salientam que a prática da agricultura urbana estimula o protagonismo social, a participação cidadã, a aquisição de habilidades pessoais e coletivas que viabiliza a formação de ambientes favoráveis à saúde.

A existência de uma "dimensão terapêutica" na agricultura urbana foi defendida por Chierrito-Arruda et al. (2018) ao estudar um jardim comunitário no Paraná relataram a percepção por parte dos usuários de um ambiente que facilita a restauração psicológica, a saúde mental, a qualidade de vida, as interações socioambientais, a produtividade, a alimentação saudável e a economia familiar. O contato com a terra na horta urbana contribui para o resgate cultural de saberes, para a construção de novos conhecimentos e para a mudança gradativa de hábitos alimentares, pois ressalta a relação alimentação-saúde-meio ambiente (Ribeiro et al., 2017).

As plantas medicinais quando inseridas nas hortas urbanas tem potencial de serem utilizadas na promoção de saúde. Em Rio Branco (AC), Siviero et al. (2012) relatam que foram identificadas 83 espécies de uso medicinal, destas 66,2\% foram classificadas como exóticas, 28,9\% estavam diretamente associadas ao uso alimentar e 16,8\% destinavam-se ao uso ornamental. Costa et al. (2015) observaram em uma Unidade Básica de Saúde ${ }^{4}$ que a prática das hortas com a presença de plantas medicinais contribui para implantação da promoção de saúde no sistema público de atendimento à população.

Nas unidades escolares a AU agroecológica contribuí no protagonismo comunitário, na participação popular e no desenvolvimento da consciência alimentar e ambiental (Ribeiro et al., 2012). A horta na escola é uma estratégia pedagógica que abre diversas possibilidades para trabalhar a relação dos estudantes com a alimentação e meio ambiente, podendo se transformar em uma iniciativa de promoção de saúde (Coelho \& Bógus, 2016; Doria et al., 2017).

\footnotetext{
${ }^{3}$ Projeto teve como objetivo promover a inserção socioeconômica, contribuir com a segurança alimentar e nutricional, promover a educação socioambiental, resgatar o saber popular, estimular a geração de trabalho e renda, e a economia solidária, com ênfase na produção agroecológica (Ribeiro et al., 2015).

${ }^{4}$ A Unidade Básica de Saúde é a estrutura inicial de acesso aos serviços públicos de saúde no Brasil, compondo o Sistema Unico de Saúde.
} 
No estado de São Paulo no município de Embu das Artes, Garcia et al. (2017) concluíram que a horta escolar proporcionou nas crianças e famílias uma reflexão sobre o ato de comer e sobre os alimentos, resultando em mudanças concretas nos hábitos alimentares. A horta escolar para além de atender objetivos relacionados às questões ambientais, alimentação e saúde pode reforçar elementos que têm sido historicamente invisibilizados como a problematização do sistema de produção agrícola hegemônico, a valorização do urbano versus a desvalorização do rural, a saúde do agricultor, entre outros (Silva et al., 2015)

A AU pode oferecer riscos à saúde humana quando realizada em ambientes contaminados ou onde ocorre a contaminação dos alimentos, por isso, deve-se ter especial preocupação com a qualidade do solo, água e do ar. A identificação de fatores de risco associados a efeitos deletérios à saúde humana é importante para fornecer uma base mais segura para a agricultura urbana (Amato-Lourenco et al., 2017). A possibilidade de contaminação de alfaces por metais-traço contidos no solo foi analisada na cidade de Belo Horizonte por Dala-Paula et al. (2018) e concluíram que os níveis de chumbo, cobre e cádmio encontrados nos vegetais indicam a produção segura.

A contaminação dos cultivos vegetais pelo ar na cidade de São Paulo foi avaliada por Amato-Lourenco et al. (2016), que encontraram em hortas comunitárias elementos químicos relacionados ao tráfego de veículos. Os autores constataram que concentrações de chumbo e cádmio em vegetais excederam os valores limite de consumo após 60 dias de exposição a poluição atmosférica em áreas mais urbanizadas da cidade. A utilização de barreiras verticais, como quebra-ventos por exemplo, é eficiente na redução dos riscos de contaminação dos cultivos vegetais por poluentes do ar (Amato-Lourenco et al., 2017).

Os artigos analisam a relação entre agricultura urbana e saúde de maneiras distintas entre pesquisas que apontavam os potenciais benefícios e outras os eventuais riscos. Destacamos o potencial da agricultura urbana ser utilizada como uma ferramenta de promoção de saúde e educação. No entanto, deve-se ter cuidado para que a implantação das hortas seja realizada em ambientes adequados e seguros para evitar a contaminação dos alimentos produzidos. Por fim, acredita-se que a agricultura urbana também pode estar relacionada aos processos de educação não formal e informal, algo não abordado na literatura analisada.

\subsection{Agricultura e Desenvolvimento Urbano}

A agricultura urbana no Brasil é um componente importante na dinâmica das cidades e possui características multifuncionais que podem beneficiar os espaços urbanos. A prática da agricultura na cidade pode ter um papel importante na melhoria do patrimônio rural tangível e intangível no contexto urbano podendo ser uma oportunidade para reforçar a identidade das pessoas e sua qualidade de vida (Villares de Carvalho \& Nella Branduini, 2017).

A dimensão econômica da AU foi abordada em Santarém por WinklerPrins e Oliveira, (2010) que apresentaram a AU como uma alternativa de renda em uma cidade onde não existem oportunidades salariais suficientes. Em Natal (RN), Locatel e Azevedo, (2010) afirmaram que a produção de alimentos obtida através da AU não serve apenas para o autoconsumo, mas como principal, e às vezes a única fonte de renda familiar. Em Imperatriz (MA), 28\% dos agricultores urbanos estudados optaram por desenvolver a atividade como meio de subsistência em razão do desemprego (Oliveira et al., 2019).

A agricultura enquanto uma prática social realizada nas cidades tende a se desenvolver de acordo com as possibilidades de ocupação do espaço urbano em concordância com a legislação vigente. Araújo (2016) afirma que é necessário ir além das funcionalidades alimentar e econômica, pois a agricultura urbana deveria ser incluída como parte do planejamento ambiental das cidades. A realização de zoneamento urbano que integre os aspectos ecológicos com o território urbanizado permitiria promover a responsabilidade da produção de alimentos e sustentabilidade ambiental nas cidades (Lemos et al., 2015). Portanto, é imprescindível que os planejadores urbanos reconheçam a importância da agricultura urbana e a incorpore nas políticas públicas como um elemento fundamental para a sustentabilidade urbana (Batitucci et al., 2019). 
Existem registros no Brasil de avanços nas legislações mais adequadas para o desenvolvimento da agricultura urbana. Em Belo Horizonte, por exemplo, o Plano Diretor, Lei n 9.959/2010 estabelece normas e condições para parcelamento, ocupação e uso do solo urbano (Araújo, 2016). Desta forma, a autora relata que no plano diretor AU é incluída como categoria possível de ser praticada em solo urbano o que, certamente, é um passo importante para o desenvolvimento das políticas públicas.

A prática da agricultura nos espaços urbanos pode gerar conflitos entre os diferentes grupos sociais e com o poder público. Em Castanhal, no estado do Pará, Ruas et al. (2014) relatam as dificuldades de pequenos pecuaristas que vivem em região periurbana e que utilizam as vias públicas para o pastoreio. Os autores reportam que por falta de legitimidade social e de normas específicas, a atividade pecuária periurbana foi perdendo espaço e deixando de ser praticada e os moradores não somente perderam a oportunidade de criar seus próprios animais, mas também tiveram a redução de suprimentos, antes oriundos da pecuária como por exemplo, carne, leite e derivados.

Com o desenvolvimento urbano surgem novas possibilidades de se praticar a agricultura nas cidades, como, por exemplo, o cultivo de plantas integrado as edificações. Sanyé-Mengual et al. (2018) estimaram o potencial de produção de alimentos e os benefícios ambientais na implantação de estufas no telhado dos edifícios comerciais em oito cidades na Europa e América do Sul, incluindo a cidade de São Carlos no Brasil. O trabalho concluiu que essa prática permitiria uma produção entre 31 a 234 toneladas de tomate por hectare, gerando um abastecimento alimentar do produto para 380 a 21.500 pessoas. Além disso, seria possível economizar entre 16 a 112 toneladas de $\mathrm{CO}^{2}$ equivalente por hectare. A integração do fluxo de água na captação da chuva no prédio para a produção agrícola garantiria a autossuficiência da água da cultura.

A integração da agricultura urbana às políticas públicas em distintas áreas do governo é um desafio para desenvolver ações concretas em prol do desenvolvimento urbano sustentável. Em Belo Horizonte, Rocha e Lessa (2009) relatam que através de políticas de segurança alimentar o governo instituiu um programa de suporte a agricultura urbana desenvolvido a partir de quatro subprojetos, os jardins comunitários, escolares, plantio de árvores em áreas comuns e oficinas de plantio em espaços alternativos. Até 2008 a cidade de Belo Horizonte possuía 44 hortas comunitárias e 60 hortas escolares, distribuiu mais de 1600 mudas de árvores frutíferas e ofereceu 62 oficinas de jardinagem em espaços alternativos, com a participação de mais de 1300 pessoas. Para o desenvolvimento de políticas públicas Araújo (2016) defende que a AU deve ser entendida como potencialmente promotora de desenvolvimento local e não apenas como uma atividade promotora de abastecimento alimentar.

A agricultura urbana também é vista como uma ação de cidadania e sugere-se que a atividade seja incluída na agenda de reforma urbana como uma ferramenta para democratizar o planejamento e a gestão do espaço urbano (Visoni \& Nagib, 2019). Para tornar o espaço urbano mais democrático, a prática da AU deve ser incluída na pauta de discussão do poder público, para aquilo que estende sobre o "direito à cidade" (Locatel \& Azevedo, 2010). No Brasil, o direito a cidade sustentável está descrito no Estatuto da Cidade e é compreendido como o direito à terra urbana, à moradia, ao saneamento ambiental, à infraestrutura urbana, ao transporte e aos serviços públicos, ao trabalho e ao lazer, para as presentes e futuras gerações (Amanajás \& Klug, 2018; Brasil, 2001). Nesse sentido, para AU tornar-se uma ferramenta de desenvolvimento local é preciso inseri-la na legislação, nos planos diretores e nas políticas públicas municipais.

\section{Conclusão}

Essa pesquisa demonstra que a agricultura urbana é realizada em todas as regiões do Brasil, apresenta características multifuncionais e é um componente importante nas relações econômicas, sociais e ecológicas das cidades.

A agricultura urbana tem potencial de contribuir na segurança alimentar e nutricional, na promoção de saúde, na educação e no ambiente urbano, contribuindo dessa forma para o desenvolvimento de cidades sustentáveis. 


\section{Agradecimentos}

Ao Programa de Bolsa de Monitoria de Pós-Graduação (PROMOP) da Universidade do Estado de Santa Catarina (UDESC) pela concessão de bolsa de Doutorado para o desenvolvimento dessa pesquisa e ao CNPq (processos n. 304018/2015 e 307376/2017-6).

\section{Referências}

Abud, G. M. B., Lopes, M. L. B., Do Socorro Silva Corrêa, R., \& Almeida, R. H. C. (2019). Urban and peri-urban agriculture: Potentialit y and limitations for the development of Benevides, Brazil. Revista Em Agronegocio e Meio Ambiente, 12(4), 1395-1416. https://doi.org/10.17765/2176-9168.2019v12n4p13951416

Amanajás, R., \& Klug, L. (2018). Direito à cidade, cidades para todos e estrutura sociocultura urbana. In Costa, M. A., Magalhães, M. T. Q., \& Favarão, C. B. (Eds.), A nova agenda urbana no Brasil: insumos para a sua construção e desafios a sua implementação (pp. 29-44). IPEA.

Amato-Lourenco, L. F., Lobo, D. J. A., Guimarães, E. T., Moreira, T. C. L., Carvalho-Oliveira, R., Saiki, M., Saldiva, P. H. N., \& Mauad, T. (2017). Biomonitoring of genotoxic effects and elemental accumulation derived from air pollution in community urban gardens. Science of the Total Environment, 575, 1438-1444. https://doi.org/10.1016/j.scitotenv.2016.09.221

Amato-Lourenco, L. F., Moreira, T. C. L., De Oliveira Souza, V. C., Barbosa, F., Saiki, M., Saldiva, P. H. N., \& Mauad, T. (2016). The influence of atmospheric particles on the elemental content of vegetables in urban gardens of Sao Paulo, Brazil. Environmental Pollution, 216 , 125-134. https://doi.org/10.1016/j.envpol.2016.05.036

Araújo, A. S. (2016). O Planejamento Urbano E Ambiental Na Construção De Cidades Sustentáveis: As hortas urbanas comunitárias em Porto, Portugal, e Belo Horizonte, Brasil. Urbana: Rev. Eletrônica Cent. Interdiscip. Estud. Cid, 8(2), 190-209.

Batitucci, T. de O., Cortines, E., Almeida, F. S., \& Almeida, A. A. de. (2019). A Agricultura em ecossitemas urbanos:um passo para a sustentabilidade das cidades. Ambiente \& Sociedade, 22, 1-20.

Brasil. (2001). LEI n. 10.257, DE 10 DE JULHO DE 2001. Regulamenta os arts. 182 e 183 da Constituição Federal, estabelece diretrizes gerais da política urbana e dá outras providências. http://www.planalto.gov.br/ccivil_03/leis/leis_2001/110257.htm

Cabannes, Y. (2012). Financing urban agriculture. Environment and Urbanization, 24(2), 665-683. https://doi.org/10.1177/0956247812456126

Castelo Branco, M., Liz, R., Alcântara, F., Martins, H., \& Hanson, J. (2011). Agricultura Apoiada pela Comunidade: poderia a experiência dos agricultores americanos ser útil para os agricultores urbanos brasileiros? Horticultura Brasileira, 29(1), 43-49. https://doi.org/10.1590/s0102-05362011000100008

Chierrito-Arruda, E., Yaegashi, S. F. R., Paccola, E. A. de S., \& Grossi-Milani, R. (2018). Percepção Ambiental e Afetividade: Vivências em uma horta comunitária. Ambiente \& Sociedade, 21.

Coelho, D. E. P., \& Bógus, C. M. (2016). Vivências de plantar e comer: A horta escolar como prática educativa, sob a perspectiva dos educadores. Saude $e$ Sociedade, 25(3), 761-771. https://doi.org/10.1590/S0104-12902016149487

Costa, C. G. A., Garcia, M. T., Ribeiro, S. M., Salandini, M. F. de S., \& Bógus, C. M. (2015). Hortas comunitárias como atividade promotora de saúde: Uma experiência em Unidades Básicas de Saúde. Ciência e Saúde Coletiva, 20(10), 3099-3110. https://doi.org/10.1590/1413-812320152010.00352015

Dala-Paula, B. M., Custódio, F. B., Knupp, E. A. N., Palmieri, H. E. L., Silva, J. B. B., \& Glória, M. B. A. (2018). Cadmium, copper and lead levels in different cultivars of lettuce and soil from urban agriculture. Environmental Pollution, 242, 383-389. https://doi.org/10.1016/j.envpol.2018.04.101

Dale, V. H., Kline, K. L., Kaffka, S. R., \& Langeveld, J. W. A. (Hans. (2013). A landscape perspective on sustainability of agricultural systems. Landscape Ecology, 28(6), 1111-1123. https://doi.org/10.1007/s10980-012-9814-4

de Medeiros, N. S., do Carmo, D. L., Priore, S. E., Santos, R. H. S., \& Pinto, C. A. (2018). Food security and edible plant cultivation in the urban gardens of socially disadvantaged families in the municipality of Viçosa, Minas Gerais, Brazil. Environment, Development and Sustainability, $21(3), 1171-1184$. https://doi.org/10.1007/s10668-017-0078-7

Doria, N. G., Coelho, D. E. P., Garcia, M. T., Watanabe, H. A. W., \& Bógus, C. M. (2017). the Experience of an Agroecological School Garden As an Interactive and Creative Health Promotion Strategy. DEMETRA: Alimentação, Nutrição \& Saúde, 12(1), 69-90. https://doi.org/10.12957/demetra.2017.23788

Eichemberg, M. T., \& Amorozo, M. C. de M. (2013). Contribuições dos quintais urbanos antigos na produção e no consumo de alimentos em Rio Claro, Sudeste do Brasil. Boletim Do Museu Paraense Emilio Goeldi:Ciencias Humanas, 8(3), 745-755. https://doi.org/10.1590/S1981-81222013000300015

Emperaire, L., \& Eloy, L. (2015). Amerindian Agriculture in an Urbanising Amazonia (Rio Negro, Brazil). Bulletin of Latin American Research, 34(1), 70-84. https://doi.org/10.1111/blar.12176

FAO. (2014). Food for the Cities. 8. http://www.fao.org/fcit/fcit-home/en/

FAO, IFAD, UNICEF, WFP \& WHO. (2020). 2020 The State of Food Security and Nutrition in the World. In FAO. FAO/ONU.

Garcia, M. T., Coelho, D. E. P., \& Bógus, C. M. (2017). Pedagogical School Gardens As a Food and Nutrition Education Strategy: Perception of Parents and Educators of Their Impact on Children'S Diets. DEMETRA: Alimentação, Nutrição \& Saúde, 12(1). https://doi.org/10.12957/demetra.2017.26407

Gondim, M. F. (2014). A travessia no tempo: Homens e veículos, da mitologia aos tempos modernos. Universidade de Brasilia. 
Harari, Y. N. (2017). Sapiens: Uma breve história da humanidade (1st ed.). L\&PM.

Hearn, A. H. (2018). Beanstalks and Trust in Chinese and Brazilian Food Systems. Journal of Latin American Geography, 17(2), 84-112. https://doi.org/10.1353/lag.2018.0023

Lemos, N. D. S., Andrade, L. M. D. S. de., \& Medeiros, V. A. S. de. (2015). Desafios do Planejamento Urbano no Brasil e seus Marcos legais sob a Ótica da Agricultura Urbana. Paranoá: Cadernos de Arquitetura e Urbanismo, 61-70. https://doi.org/10.18830/issn.1679-0944.n14.2015.16988

Locatel, C. D., \& Azevedo, F. F. de. (2010). Gestão do Território e a prática da agricultura urbana na cidade de Natal (RN-Brasil). Scrita Nova, XIV(331), 1-13.

Lovell, S. T. (2010). Multifunctional urban agriculture for sustainable land use planning in the United States. Sustainability, 2(8), 2499-2522. https://doi.org/10.3390/su2082499

Malta, D. C., Morais Neto, O. L., da Silva, M. M. A., Rocha, D., de Castro, A. M., dos Reis, A. A. C., \& Akerman, M. (2016). Política Nacional de Promoção da Saúde (PNPS): Capítulos de uma caminhada ainda em construção. Ciência e Saúde Coletiva, 21(6), 1683-1694. https://doi.org/10.1590/141381232015216.07572016

Mazoyer, M., \& Roudart, L. (2010). História das agriculturas no mundo: do neolítico à crise contemporânea. In Editora Unesp. São Paulo. NEAD.

Moraes, J. G. de, Breda, M. O., Alves, R. R. N., \& Alves, Â. G. C. (2017). A percepção de agricultores urbanos sobre pragas e plantas cultivadas em uma área metropolitana do Recife. Biotemas, 30(1), 99. https://doi.org/10.5007/2175-7925.2017v30n1p99

Moura, J. A., Ferreira, W. R., \& Lara, L. L. (2013). Agricultura Urbana e Periurbana. Mercator, 12(27), 69-80. https://doi.org/10.4215/rm2013.1227.0005

Oliveira, E. A. A. Q., Nunes, L. L. M., \& Moraes, M. B. de. (2019). Características Socioeconômica e ambiental da Agricultura Urbana em Imperatriz-MA. Redes, 24(3), 241-271. https://doi.org/10.17058/redes.v24i3.14105

ONU. (2012). Fatos sobre as cidades - Rio + 20. Rio+20. O Futuro Que Queremos. http://www.onu.org.br/rio20/cidades.pdf

ONU. (2017). Nueva Agenda Urbana. ONU. http://urbanhabitat.com.ar/data/Planear el Barrio.pdf

ONU. (2019). World Populations Prospects 2019: Highlights. ONU. https://population.un.org/wpp/Publications/Files/WPP2019_Highlights.pdf

Rayol, B. P., Do Vale, I., \& Miranda, I. S. (2017). Tree and palm diversity in homegardens in the Central Amazon. Agroforestry Systems, 93(2), 515-529. https://doi.org/10.1007/s10457-017-0144-Z

Ribeiro, S. M., Azevedo, E. de, Pelicioni, M. C. F., Bógus, C. M., \& Pereira, I. M. T. B. (2012). Agricultura Urbana Agroecológica - Estratégia De Promoção Da Saúde E Segurança Alimentar E Nutricional. Rev Bras Promoção Saúde, 25(3), 381-388.

Ribeiro, S. M., Bógus, C. M., \& Watanabe, H. A. W. (2015). Agricultura urbana agroecológica na perspectiva da promoção da saúde. Saúde e Sociedade, 24(2), 730-743. https://doi.org/10.1590/S0104-12902015000200026

Ribeiro, S. M., Franco, J. V., Garcia, M. T., Bógus, C. M., \& Watanabe, H. A. W. (2017). Recovery of Healthy and Sustainable Practices Through Experiences of Agroecology and Urban Agriculture. DEMETRA: Alimentação, Nutrição \& Saúde, 12(4), 1113-1132. https://doi.org/10.12957/demetra.2017.28751

Rocha, C., \& Lessa, I. (2009). Urban governance for food security: The alternative food system in Belo Horizonte, Brazil. International Planning Studies, 14(4), 389-400. https://doi.org/10.1080/13563471003642787

Rodrigues, C. de O., Lopes, M. L. B., Rebello, F. K., \& Corrêa, R. do S. da S. (2020). Caracterização socioeconômica dos sistemas de Agricultura Urbana e Periurbana adotados no Município de Irituia, Pará, Brasil. Research, Society and Development, 9(7), 1-21.

Ruas, R. M. S., Guerra, G. A. D., Furtado, D. C., \& Assis, W. S. de. (2014). Pressões e oposições contra pequenos criadores familiares de gado bovino leiteiro da zona periurbana do Município de Castanhal (Pará, Brasil - Amazônia Oriental). Mundo Agrario, 15(30).

Santos, C. J. S. E. (2017). O uso de geotecnologias abertas na disciplina Geografia Agrária: experiências na espacialização e diagnóstico da ocorrência da agricultura urbana no bairro Santa Lúcia, Maceió/AL. Diversitas Journal, 2(1), 14. https://doi.org/10.17648/diversitas-journal-v2i4.366

Sanyé-Mengual, E., Martinez-Blanco, J., Finkbeiner, M., Cerdà, M., Camargo, M., Ometto, A. R., Velásquez, L. S., Villada, G., Niza, S., Pina, A., Ferreira, G., Oliver-Solà, J., Montero, J. I., \& Rieradevall, J. (2018). Urban horticulture in retail parks: Environmental assessment of the potential implementation of rooftop greenhouses in European and South American cities. Journal of Cleaner Production, 172, 3081-3091. https://doi.org/10.1016/j.jclepro.2017.11.103

Silva, E. C. R., Da Fonseca, A. B. C., Dysarz, F. P., \& Reis, E. J. (2015). Hortas escolares: possibilidades de anunciar e denunciar invisibilidades nas práticas educativas sobre alimentação e saúde. Alexandria: Revista de Educação Em Ciência e Tecnologia, 8(1), 265-288. https://doi.org/10.5007/1982$5153.2015 \mathrm{v} 8 \mathrm{n} 1 \mathrm{p} 265$

Silva, L. S. E., \& Travassos, L. (2008). Problemas ambientais urbanos: desafios para a elaboração de políticas públicas integradas. Cadernos Metrópole, 1(19), 27-47. https://doi.org/10.1590/8708

Siqueira, D. P., Espósito, M. P., \& Souza, B. C. L. de. (2019). Direito à alimentação e os direitos da personalidade: da previsão à concretização desse direito sob a perspectiva do acesso à justiça. Revista de Constitucionalização Do Direito Brasileiro, 2(2), 1-28. https://doi.org/10.33636/reconto.v2n2.e025

Siviero, A., Delunardo, T. A., Haverroth, M., Oliveira, L. C. de., \& Mendonça, Â. M. S. (2011). Cultivo de espécies alimentares em quintais urbanos de Rio Branco, Acre, Brasil. Acta Botanica Brasilica, 25(3), 549-556. https://doi.org/10.1590/s0102-33062011000300006

Siviero, A., Delunardo, T. A., Haverroth, M., Oliveira, L. C., \& Mendonça, A. M. S. (2012). Plantas medicinais em quintais urbanos de Rio Branco, Acre. Revista Brasileira de Plantas Medicinais, 14(4), 598-610. https://doi.org/10.1590/S1516-05722012000400005 
Research, Society and Development, v. 10, n. 5, e30610514926, 2021

(CC BY 4.0) | ISSN 2525-3409 | DOI: http://dx.doi.org/10.33448/rsd-v10i5.14926

Urrútia, G., \& Bonfill, X. (2010). PRISMA declaration: A proposal to improve the publication of systematic reviews and meta-analyses. Medicina Clinica, 135(11), 507-511. https://doi.org/10.1016/j.medcli.2010.01.015

Veiga, J. E. da. (2007). O desenvolvimento agrícola: uma visão histórica (2nd ed.). Edusp.

Villares de Carvalho, I., \& Nella Branduini, P. (2017). Patrimonio y agricultura urbana en Recife: Análisis y directrices para el barrio de Várzea. Revista Urbano, 20(36), 30-41. https://doi.org/10.22320/07183607.2017.20.36.03

Visoni, C., \& Nagib, G. (2019). Reappropriating urban space through community gardens in Brazil. Field Actions Science Report, 2019(Special Issue 20), 8891.

Wandscheer, E. A. R., \& Medeiros, R. M. V. (2012). Agricultura Urbana Em Belém Do Pará: Atividade Produtiva, Dinâmicas Socioeconômicas E Organização Espacial. Geo UERJ, 1(23), 192-222. https://doi.org/10.12957/geouerj.2012.3707

WinklerPrins, A., \& Oliveira, P. S. de S. (2010). Urban agriculture in Santarém, Pará, Brazil: diversity and circulation of cultivated plants in urban homegardens Boletim Do Museu Paraense Emílio Goeldi. Ciências Humanas, 5(3), 571-585. https://doi.org/10.1590/s1981-81222010000300002 